\title{
An Assessment of PR and HC controlled Bootstrap Converter Fed SVM Inverter based Induction motor drive
}

\author{
Lavanya $\mathrm{R}^{1}$, Jayalakshmi $\mathrm{V}^{2}$ \\ \{maillavanya83@gmail.com, jayas 1979@yahoo.co.in
}

\begin{abstract}
Research Scholar, Bharath Institute of Higher Education and Research, Chennai ${ }^{1}$
Associate Professor, Bharath Institute of Higher Education and Research, Chennai ${ }^{2}$
\end{abstract}

\begin{abstract}
This task depends on bootstrap converter with SVM inverter. This work is wanted to improve the speed-guideline of bootstrapped converter-inverter took care of Induction Motor drive (BSCSVM-IIM) using Hysteresis Control. This work oversees assessment among PR-PR and PI-hysteresis controlled BSCSVM-IIM frameworks. The BSC is recommended among rectifier and SVMI to upgrade the voltage-pick up .HC is proposed to improve-the-dynamic reaction of BSC-SVMIIM. The goal of this work is to improve the reaction of BSC-SVM-IIM framework utilizing appropriate regulator in closed-loop. Open loop BSCSVM-IIM framework with disturbance, closed two loop PR-PRC and PI-HC based BSCSVM-IIM frameworks are formed; shown and simulated using Simulink and their results are presented. Assessment is done regarding settling time and SSE. The assessments exhibit the unmatched execution of controlled-BSC-SVMIIM framework. The proposed framework has inclinations like minimal consonant substance and quick reaction. After reenactment examines, some trial aftereffects of-BSC-SVM-IIM are given to check the adequacy of the proposed converters.
\end{abstract}

Keywords: BSC-SVMIIM, SVM inverter, PR-PR and PI-hysteres, Bootstrap Converter Fed SVM Inverter.

\section{Introduction}

Presently a-days, research is centered around Multi Level Inverters (MLI's), on the grounds that they can produce voltage waveforms with less contortion than customary inverters dependent on 2 level geographies. The upsides of fell MLIs are unmistakable for engine drives and for the utility applications. Due to the incredible interest on medium voltage highpower inverters, the cascaded inverters has gained more interest. As the quantity of levels expands, the integrated yield waveform has more advances delivering a flight of stairs wave that moves toward the ideal waveform

Multiplication of 3phase diode and S.C.R. connect rectifiers for D.C. power -supplies and as front-endconverters for inverter -based- applications, for example, customizable speed -drives and uninterruptible force supply, has brought about genuine symphonious, receptive force, gleam and reverberation issues in modern applications and in transmission/appropriation frameworks. A voltage mutilation because of current-harmonics is a significant issue for utilities. PQissues likewise incorporate uneven and sub-simultaneous recurrence flows which add to voltage hangs and floods, and are the most widely recognized reasons for 'disturbance' stumbling of the movable speed drives.

YongKeun and JongKwang proposed examination of bootstrap circuit activity with a modified PWMdrive plot for a 3phase inverter which is for a brushless dc engine drive[1-2]. A bootstrap circuit for working the high-side protected door bipolar semiconductor of a 3phase inverter for a brushless-dc engine drive was examined hypothetically to maintain a strategic distance from under voltage lockout by limiting the release of a boot-strap capacitor (BSC). Madhusoodhanan proposed a plan and assessment for medium voltage converter relevance's by providing a separated entryway driver power supply. The business gate-drivers were accessible upto $6.5 \mathrm{kV}$ IGBTs. With the advances in the $\mathrm{SiC}$, power gadgets evaluated past $10 \mathrm{kV}$ were being investigated. A medium voltage power converters are used in these gadgets. Business gatedrivers appraised for large- voltages were not accessible. These force gadgets had extremely high $\mathrm{dv} / \mathrm{dts}$. High dv/dt acquire difficulties in the entryway driver-design[3]. Yutian explained improved bootstrap strategies for fueling gliding door drivers of flying-capacitor staggered 
converters and mixture exchanged capacitor -converters. By utilizing the inalienable properties of staggered converters, these techniques can beat the restriction of traditional boot-strap strategy and make it conceivable to move ground-referred to capacity to the entirety of the coasting -switches[4].

Lei and Liu set a logical technique to assess and plan crossover exchanged capacitor and staggered converters. This explored the utilization of staggered transformation in dc--dc-applications that require an enormous voltage change. A strategy that can fill in as a manual for think about and plan staggered geographies for enormous transformation proportion applications was introduced. The proposed technique kept the conduction misfortune and exchanging misfortune consistent across the various converters[5].

Zhu and Liu focused on the Low-voltage-stress BBC with a high-voltage change pick up. The ordinary BBC had the benefits of straightforward structure, minimal effort, and the capacity to accomplish both voltage venture all over. Be that as it may, because of the negative effects of the parasitic boundaries of the gadget, the voltage change gain of the customary buck-help converter was enormously restricted. Pouladi and Farzanehfard actualized a Singleswitch delicate exchanging LED driver appropriate for battery-worked systems[6-8].

Another LED-driver circuit was given dependent on the buck converter coupled inductors for car relevance's. Delicate exchanging condition was accommodated the switch by means of a uninvolved full circuit joining the spillage inductance of the coupled inductors. Sun and Dai proposed Multimode smooth exchanging methodology for dispensing with the operational no man's land in non-reversing buck-boost converter. The presence of operational no man's land during mode exchanging in non-upsetting buck-help converter brings about wavering of the yield voltage and flimsiness of the frame work[9].

The system and impact of the operational dead land were examined, and a multimode smooth exchanging control methodology which can totally dispense with the operational dead land was proposed. Progressed 4modeadjustment based 4switch non-transforming BBC activity was presented in[10].

Yang explained Analysis demonstrating and execution of an exchanging bi-directional BBC dependent on electric-vehicle cross breed energy stockpiling for V.2.G framework. Battery worked delicate exchanging full BBLED driver with single attractive component recommended in[11-12]. Another circuit plan of 2switch buck-boost converter was introduced by Jung. A traditional 2switch buck-support (TSBB) converter can work in buck, lift, and buck-boost modes. This

presented another geography for a two switch buck-help converter with similar activity modes. In any case, the proposed TSBB converter had less conductions and exchanging parts than the ordinary TSBB converter, which lessens the force losses[13].

Precise induction of dead land end procedures for the non-transforming coordinated $\mathrm{BBC}$ was presented by Zhang.Bidirectional 3-stage DAC converter with implanted DDC converter and transporter based PWM procedure was recommended by Wang. Katherine proposed propels in framework associated PV-power-transformation frameworks. Applications in environmentally friendly power and its control (parc) - corresponding full regulator for semi converter 3phase VSI took care of IMD to upgrade time responses[14-17]. This exertion manages closed-loop semi converter3 stage acceptance engine drive(SCTPIMD) utilizing PI, FOPID and PRregulator. This exertion proposed PR regulator for SCTPIMD. PR-Fuzzy control improvement of doubly took care of enlistment generator during framework issues was proposed by Mohammad Reza[18-19].

Vector control strategy for IMD dependent on hysteresis regulator and pi regulator near examination was introduced by Shiny[20]. Other technique depended on SVM calculation. Query table DTC technique experienced high waves in force, motion and current just as shifting exchanging recurrence. Then again, SVM based DTC creates equivalently low force, transition and current waves and exchanging recurrence was looked after steady. Tangle at Hysteresis -current control of IMdrives utilizing dSPACE-DSPcontroller[21-22]. Hysteresis current control was moderately a straightforward strategy for PWM procedure with relatively great current circle reaction. This work introduced the equipment usage of hysteresis current control for vector controlled acceptance engine drives utilizing dSPACE-DSP. The examination was centered around the impact of hysteresis data transmission to the engine current quality.

\section{Research -Gap}

The exceeding effort doesn't include the simulation of PI-hysteresis controlled BSCSVM-IIM frameworks. Hence, this work deal with the simulation of PI-hysteresis controlled BSCSVM-IIM frameworks 


\section{Proposed System}

BlockDiagram of PRC-PRcontrolled-BSC-SVMI-system is outlined in Figure 1. Speed of IMis sensed and it is evaluated -with the reference-speed to get speedError (SE). This SE is directed to a PR-PRcontroller. The 'yield of PRC' is used to obtain reference current. The reference current is compared with actual-current and the currenterror is applied to a PRC-PR. The output of current PRC is used to adjust the Pulse-Width (PW) of BSC.

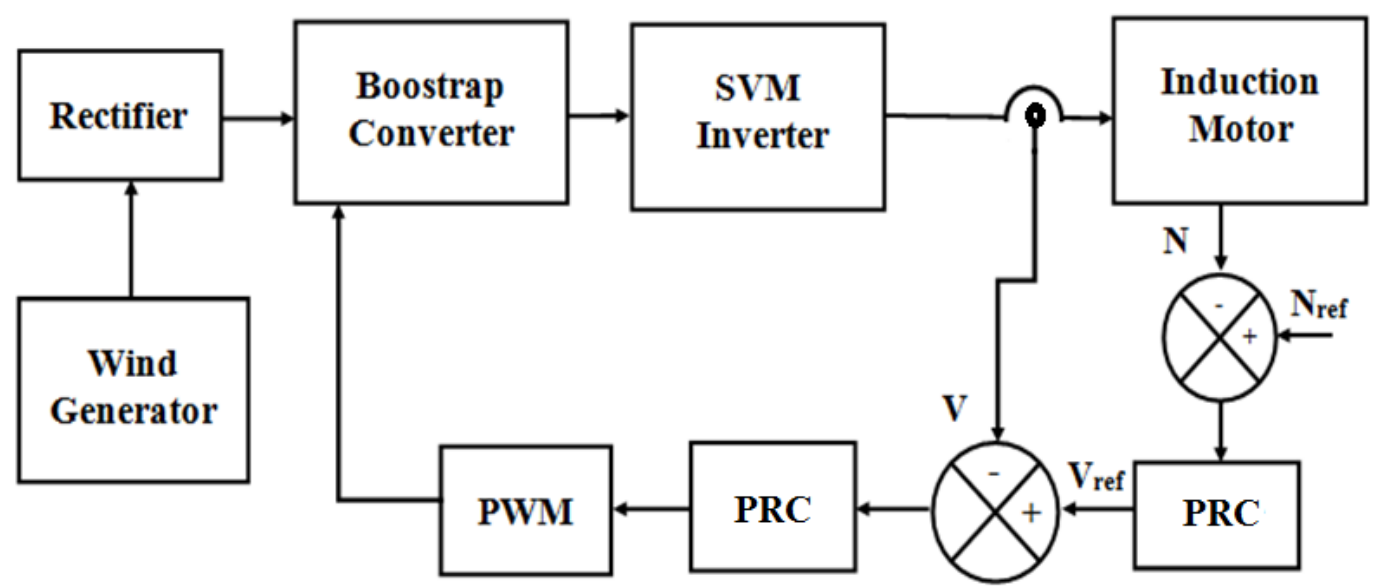

Figure 1: The Block Diagram of PRC-PR controlled closed two loop Bootstrap converter with SVM inverter

Block-Diagram of PI-Hysteresiscontrolled BSC-SVMIsystemis delineated in Figure 2.

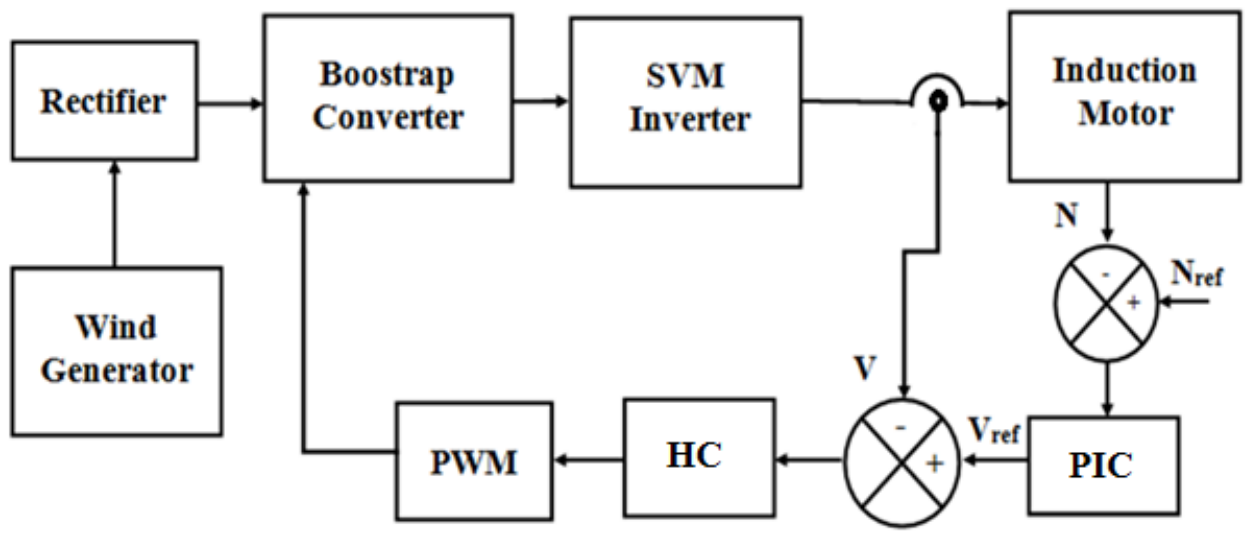

Figure 2: The Block Diagram of PIC-HC closed two loop Bootstrap converter with SVM inverter 


\section{Simulation Results}

The designed converter was first-simulated using MATLAB and then built in the laboratory to authenticate the analysis, design, \&enactment of the converter. Open loop BSCSVMIIM with load disturbanceis delinated in Figure 3.

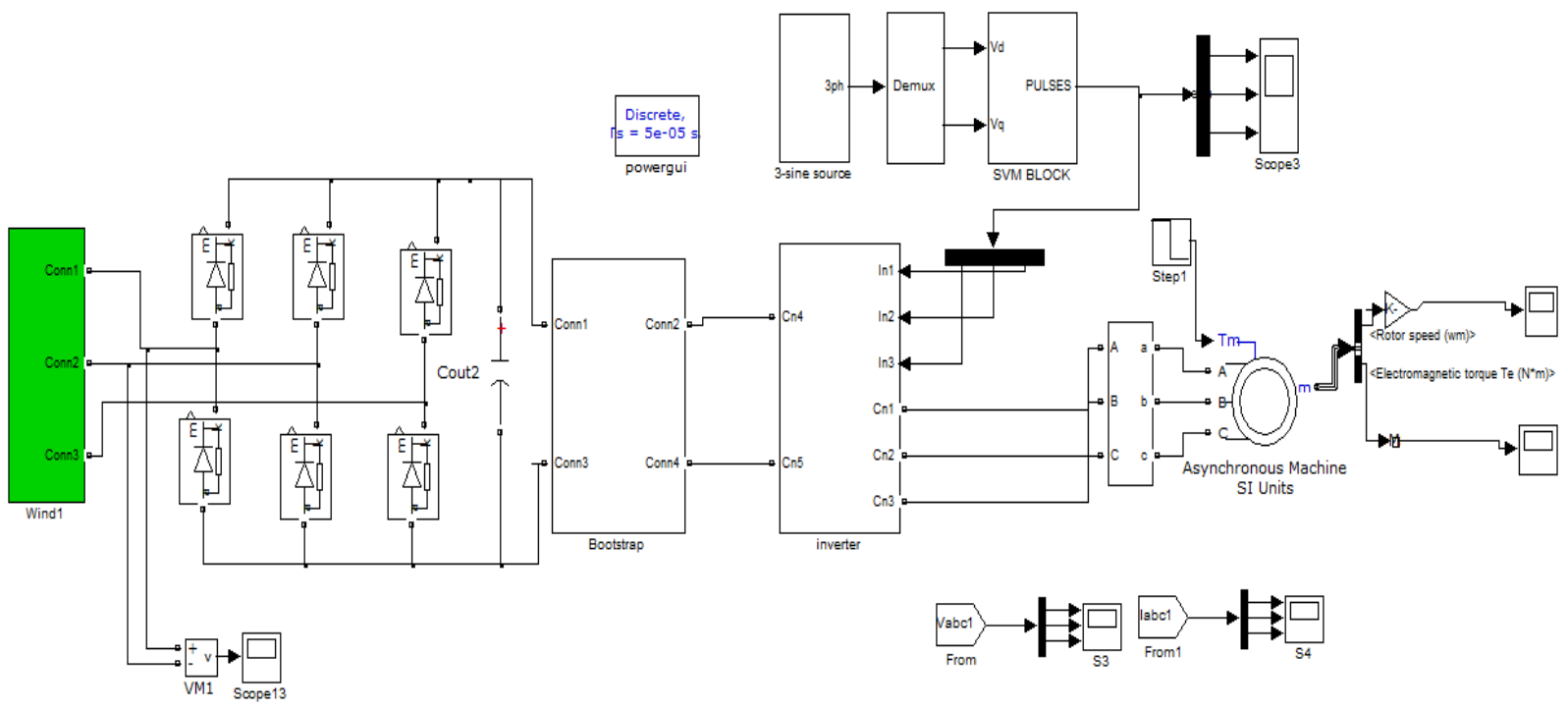

Figure 3: Circuit-diagram -of open looop BSCSVMIIMwith load disturbance

Input-voltage of BSCSVMIIM is shown in Figure 4 and the input voltage is $70 \mathrm{~V}$. The Circuit diagram of bootstrap converter is outlined in Figure 5.

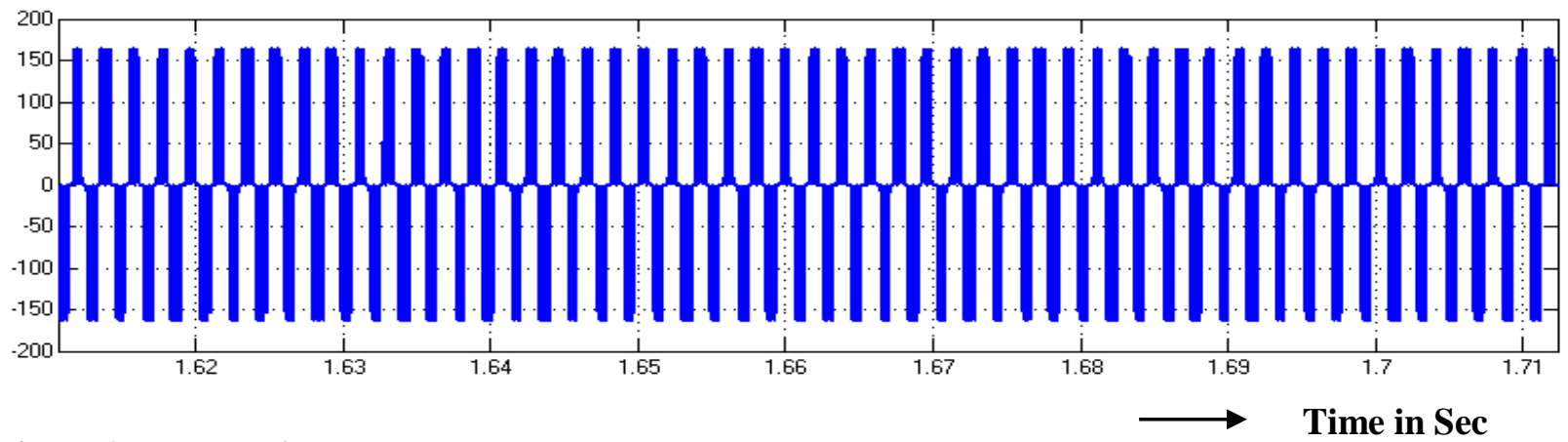

Figure4: Input voltage 


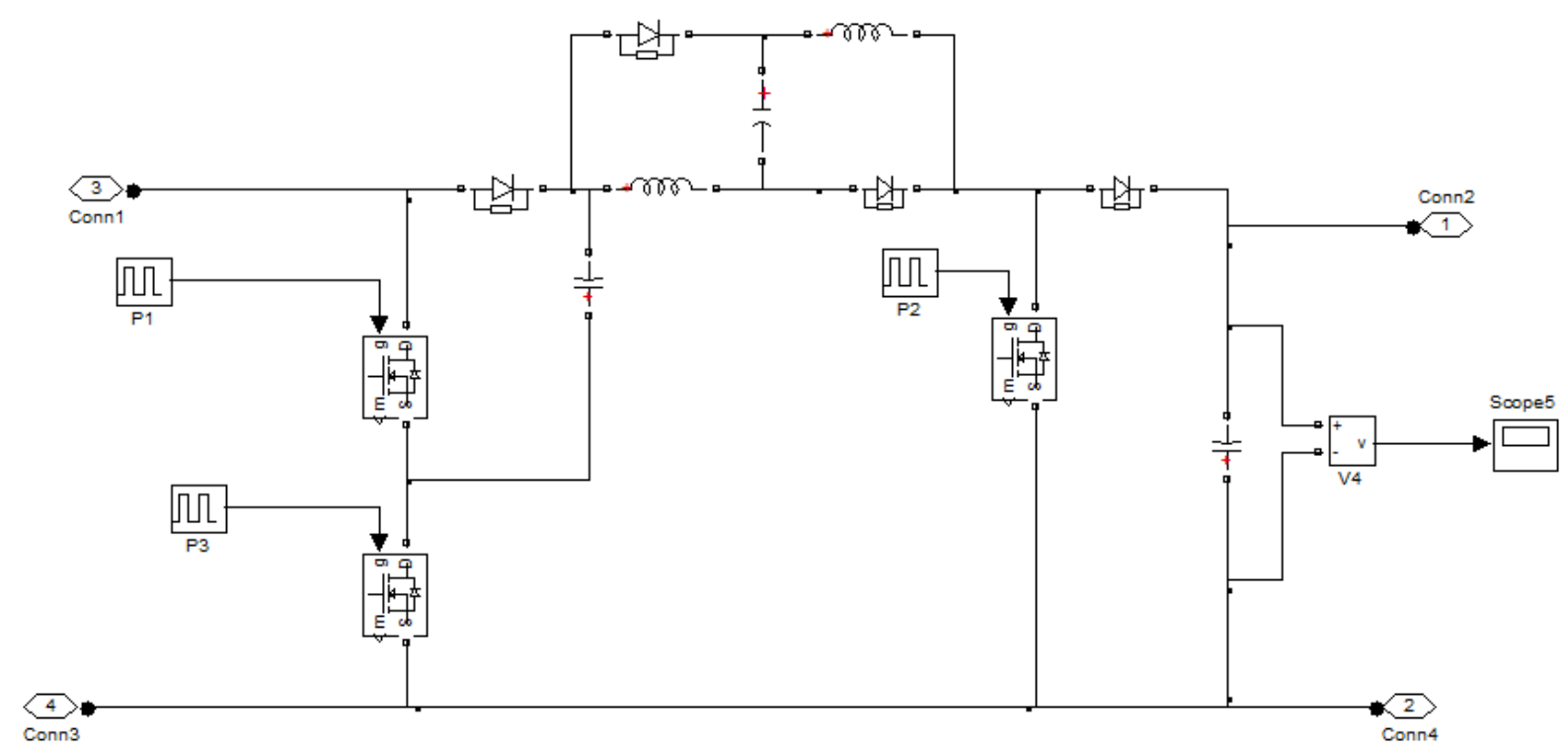

Figure5: Circuit diagram of bootstrap converter

Voltage across bootstrap converter with Bootstrap converter with SVM is presented here. Voltage across bootstrap converter is value is 410 Volts. Voltage across the motor load is 450 Volts and it is indicated in Figure 7.

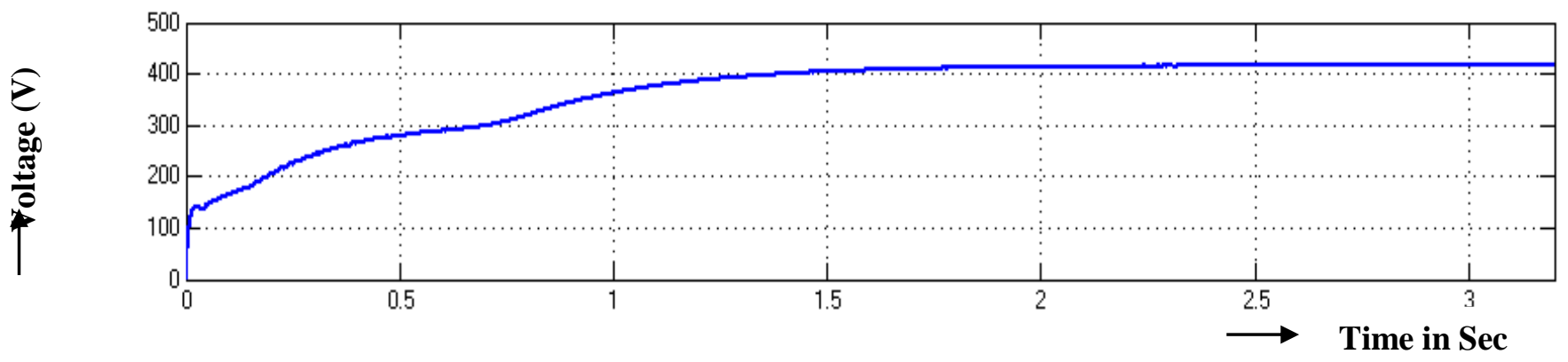

Figure6: Voltage across bootstrap converter

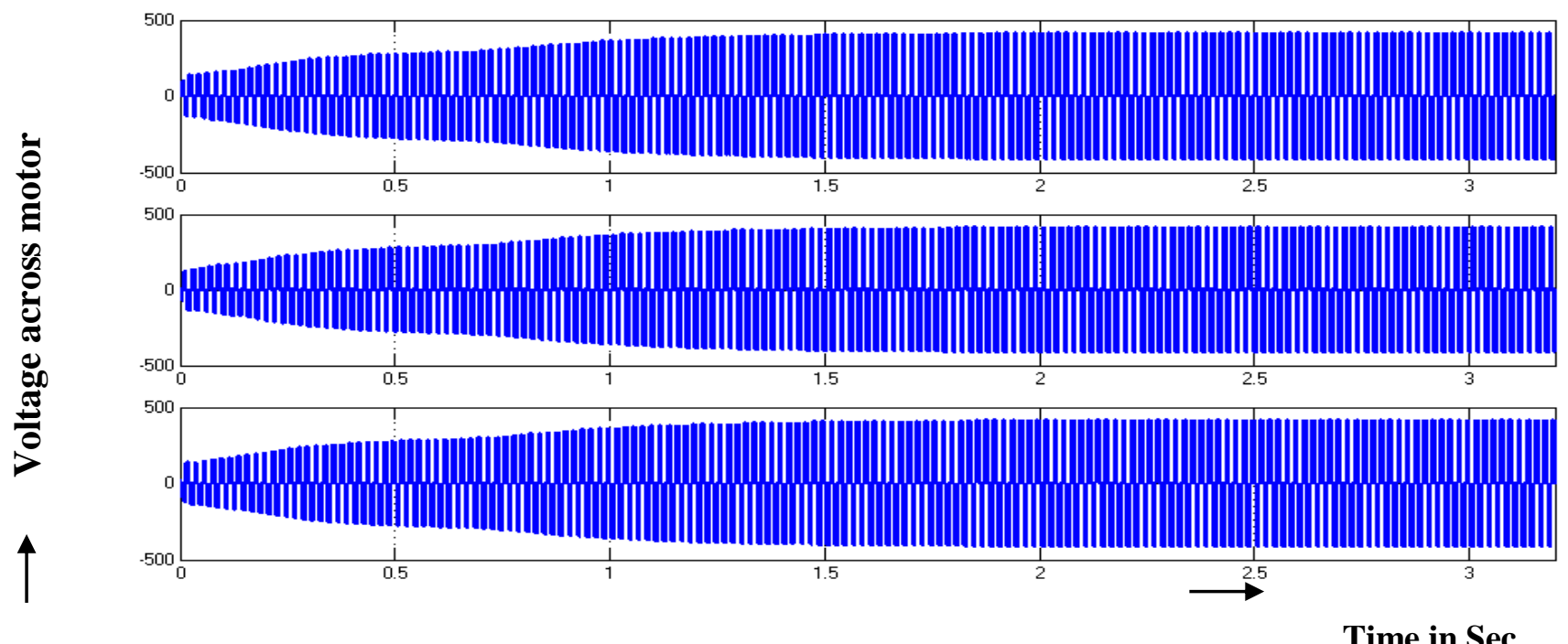

Time in Sec 
Figure7: Voltage across motor load

Motor speedwithBootstrap converter and SVM is indicated in Figure 8. The value of Motor speed 1300 RPM.

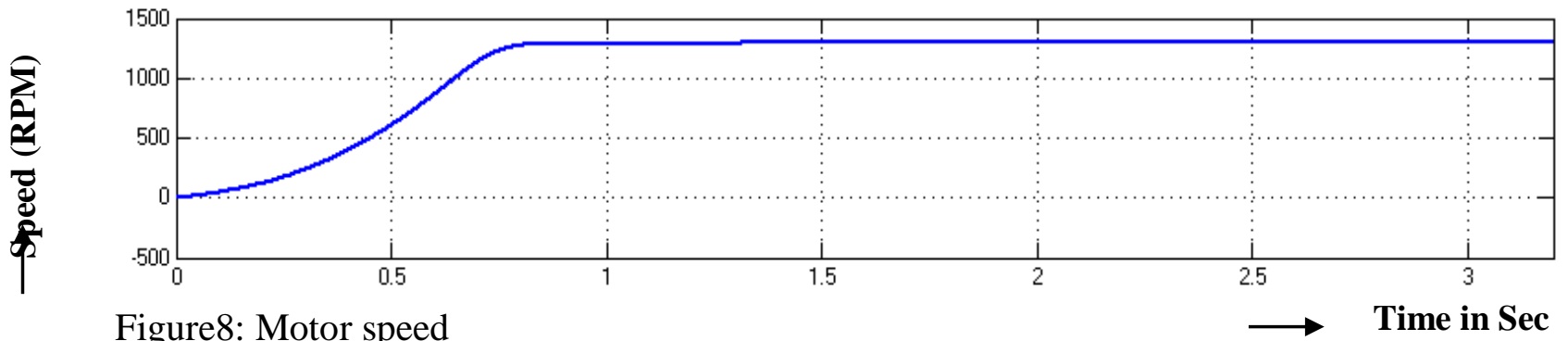

The Motor speed ofBootstrap converter with SVM is sketched in Figure 9. The value of Motor speed is 1294 RPM. MotorTorqueofBoot-strap converter along with SVM is sketched in Figure 9.The value of Motor Torqueis $1 \mathrm{~N}-\mathrm{m}$.
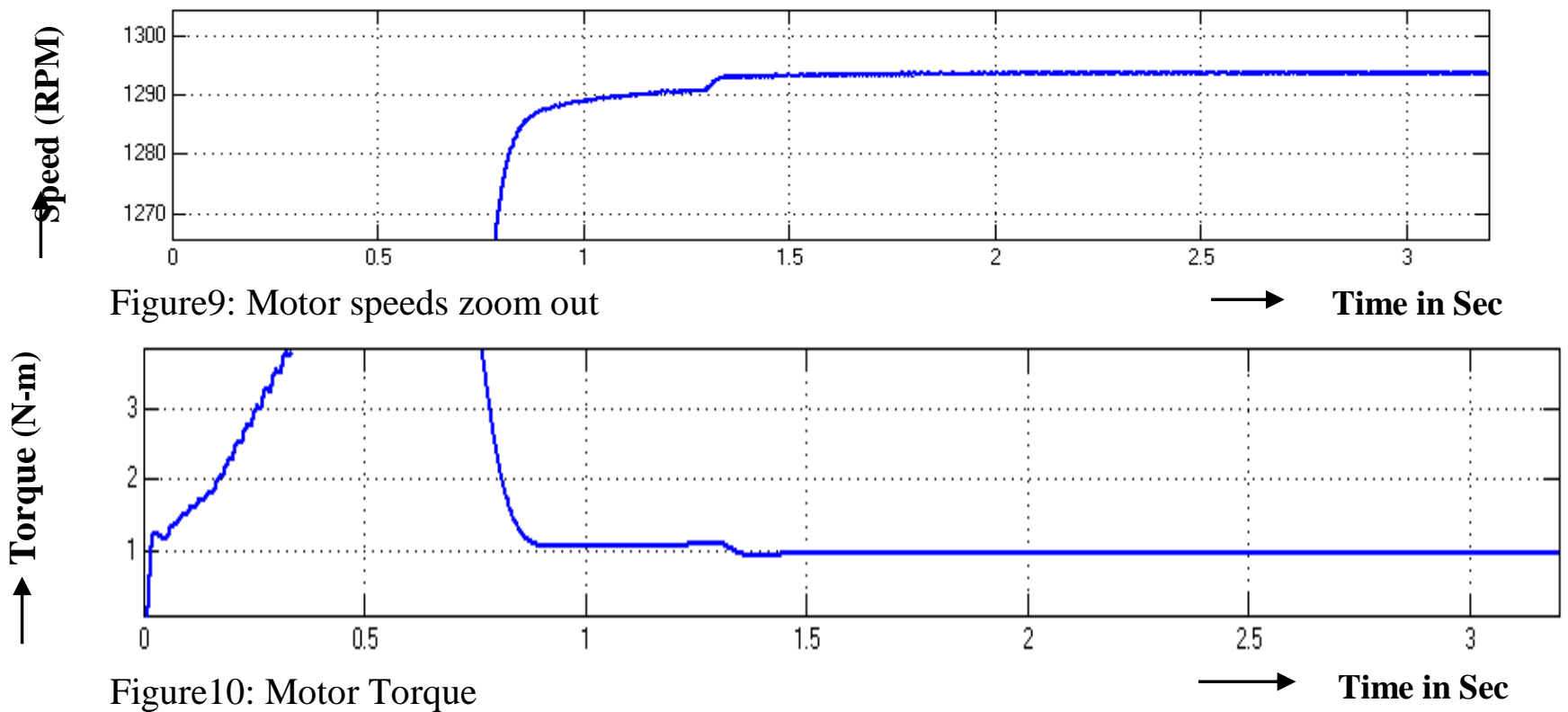

Circuit-diagramof PR-PRcontrolled closed two loop Bootstrap converter with SVM inverteris appeared in Figure 11. 


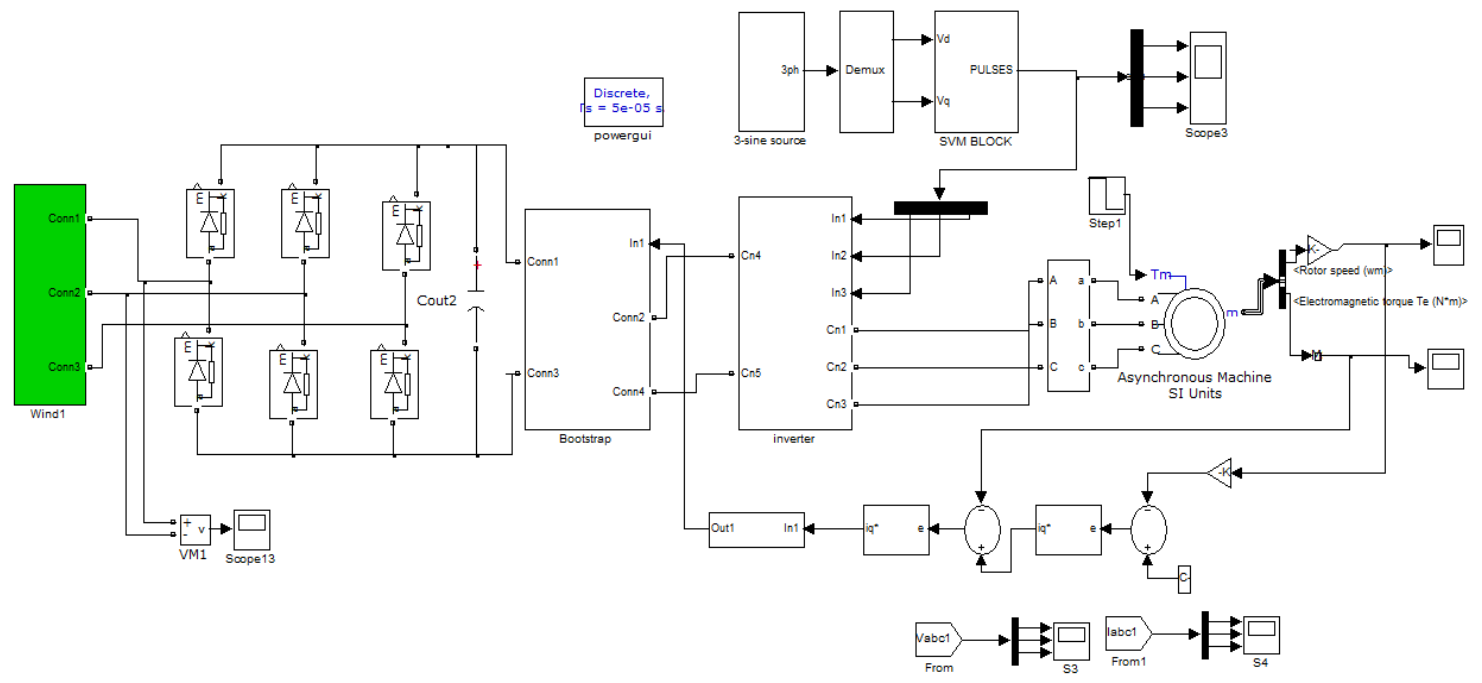

Figure 11: Circuit diagramof PR-PR controlled closed two loop Bootstrap converter with SVM inverter

Input voltageofBSCSVMIIM is outlined in Figure 12. The value of input voltage is 175 Volts. Circuit diagram of boot-strap converter is shown in Figure 13.

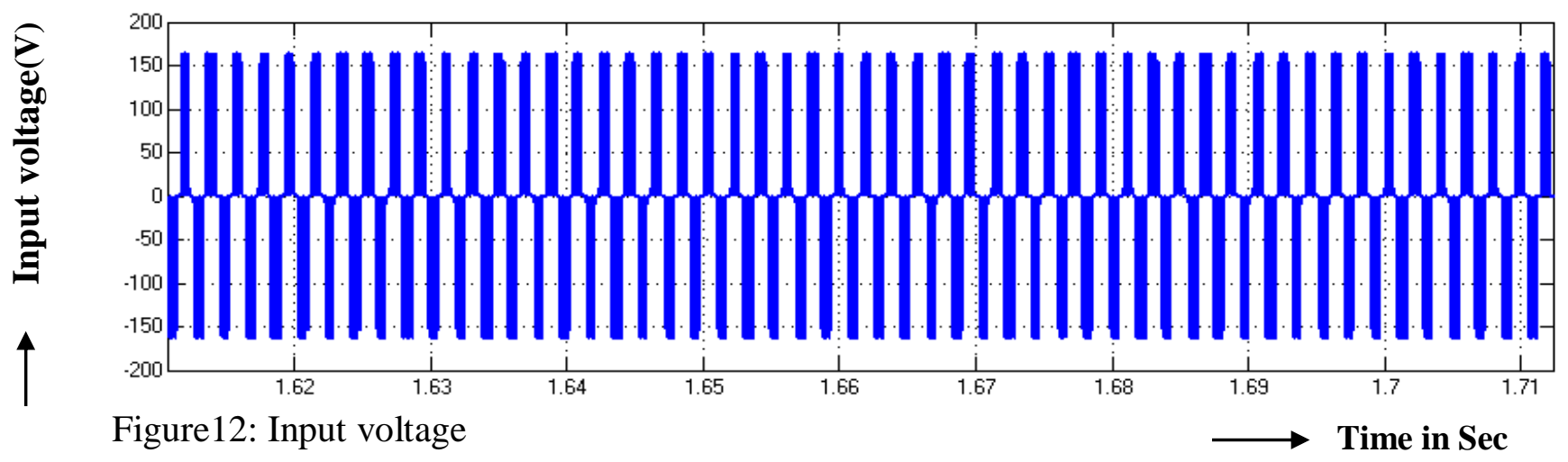




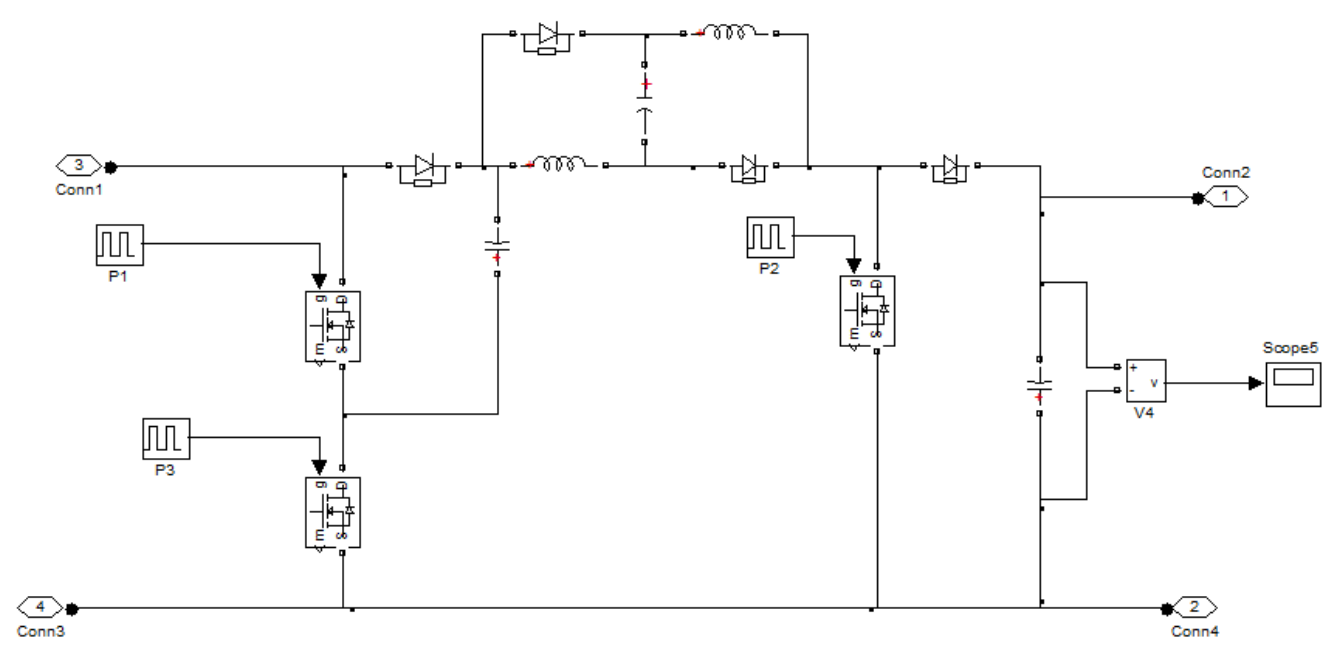

Figure 13: Circuitdiagram of bootstrap converter

The Voltage across boot-strap converter and the motor load are figured in Figure 14 and 15. The Voltage across bootstrap converter is 399 Volts and Voltage across motor load value is 400 Volts.
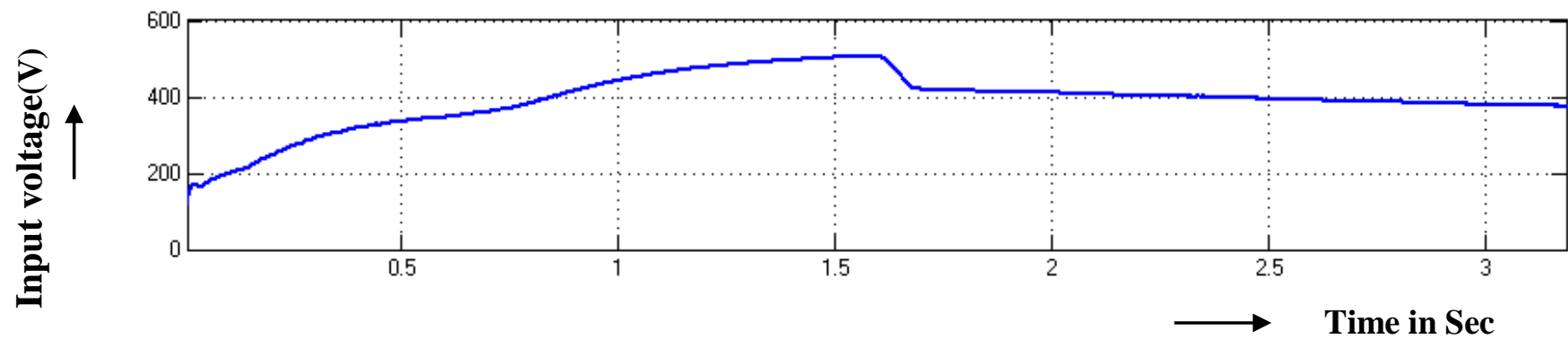

Figure14: Voltage across boot-strap converter

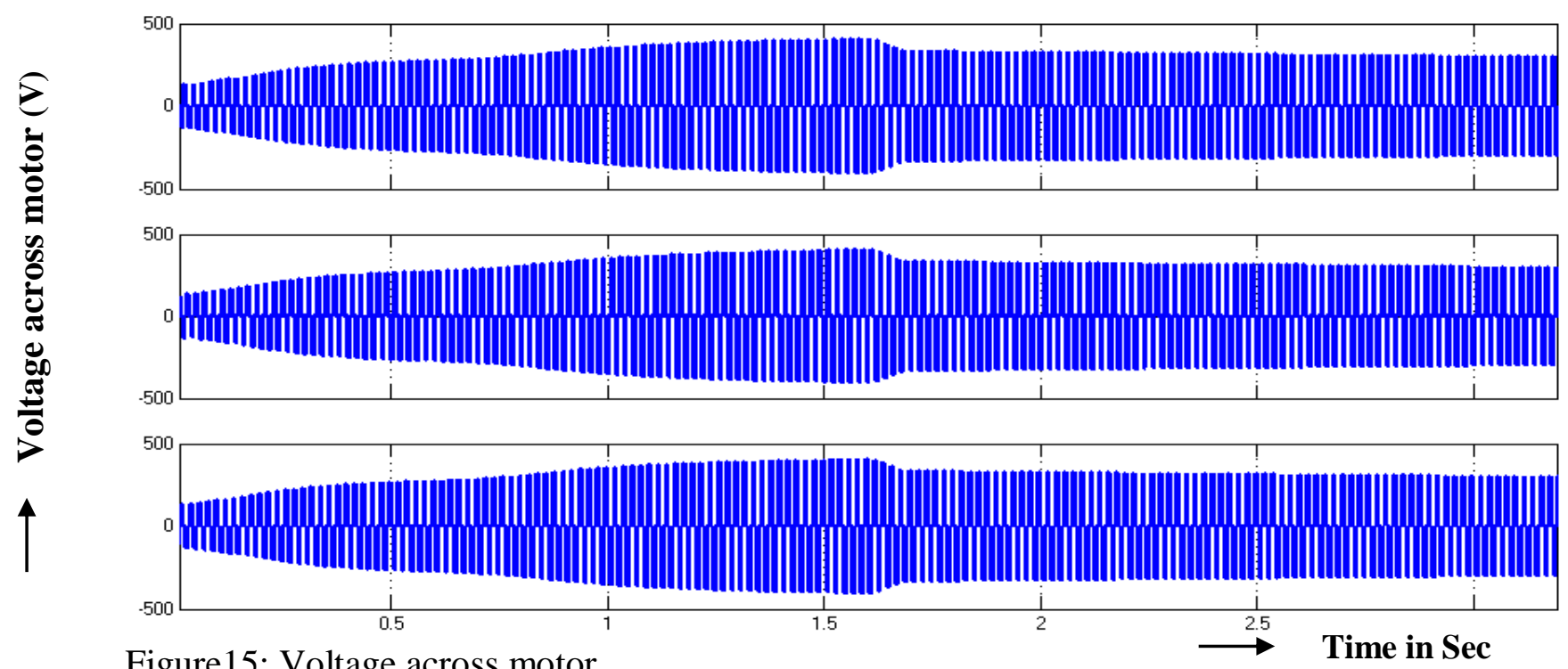

Figure15: Voltage across motor

Motor speedofBSCSVMI-IMis 1450 RPM and is appeared in Figure 16. Motor speed zoom out ofBSCSVMI-IM is 1289 RPM and is appeared in Figure 17. The Motor Torqueis 0.9 $\mathrm{N}-\mathrm{m}$ is sketched in Figure 18. 


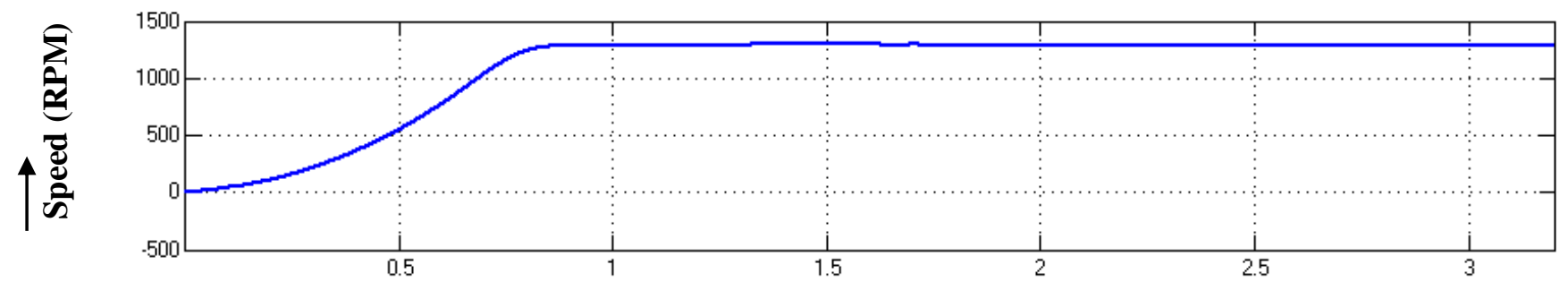

Figure16: Motor speed
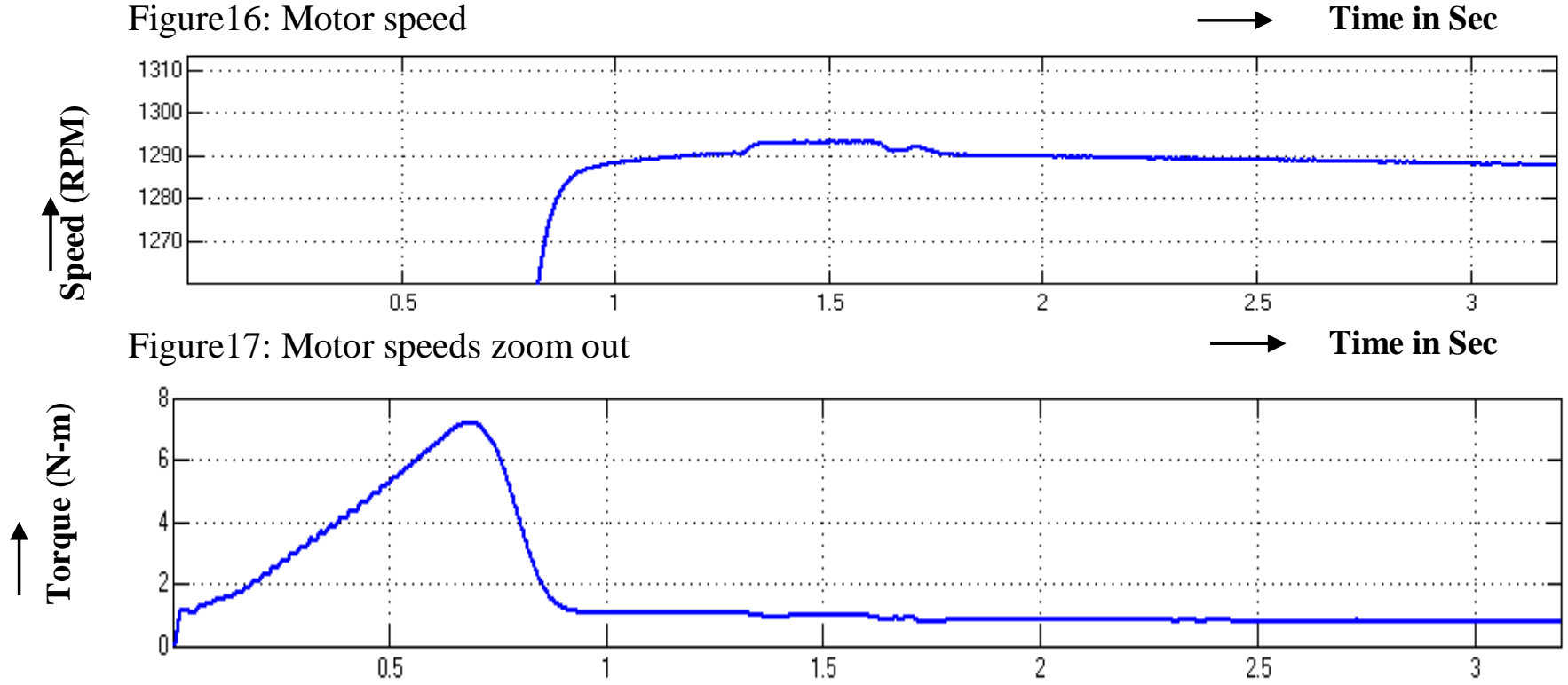

Figure18: Motor Torque

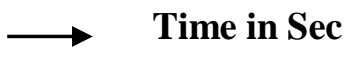

Circuit-diagramof PI-Hysteresis controlled closed two loop Bootstrap converter with SVM inverter is appeared in Figure 19.

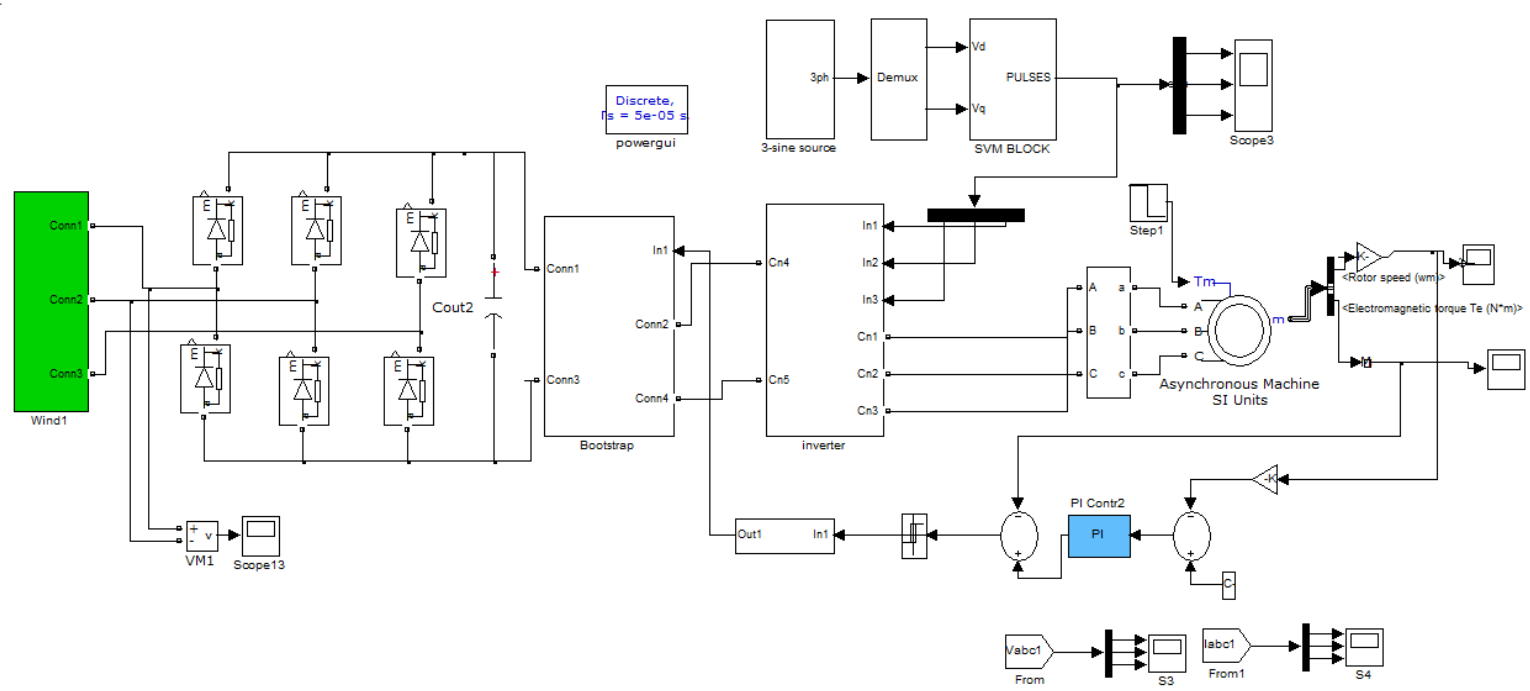

Figure 19: Circuit diagram of PI-Hysteresis controlled closed two loop Bootstrap converter with SVM inverter 
Input voltageofclosed two loopBSCSVMIIM with PI-Hysteresis controller is sketched in Figure 20. The value of input voltage is 170 Volts. The Circuit diagram of boot-strapconverter is ketched in Figure 21.
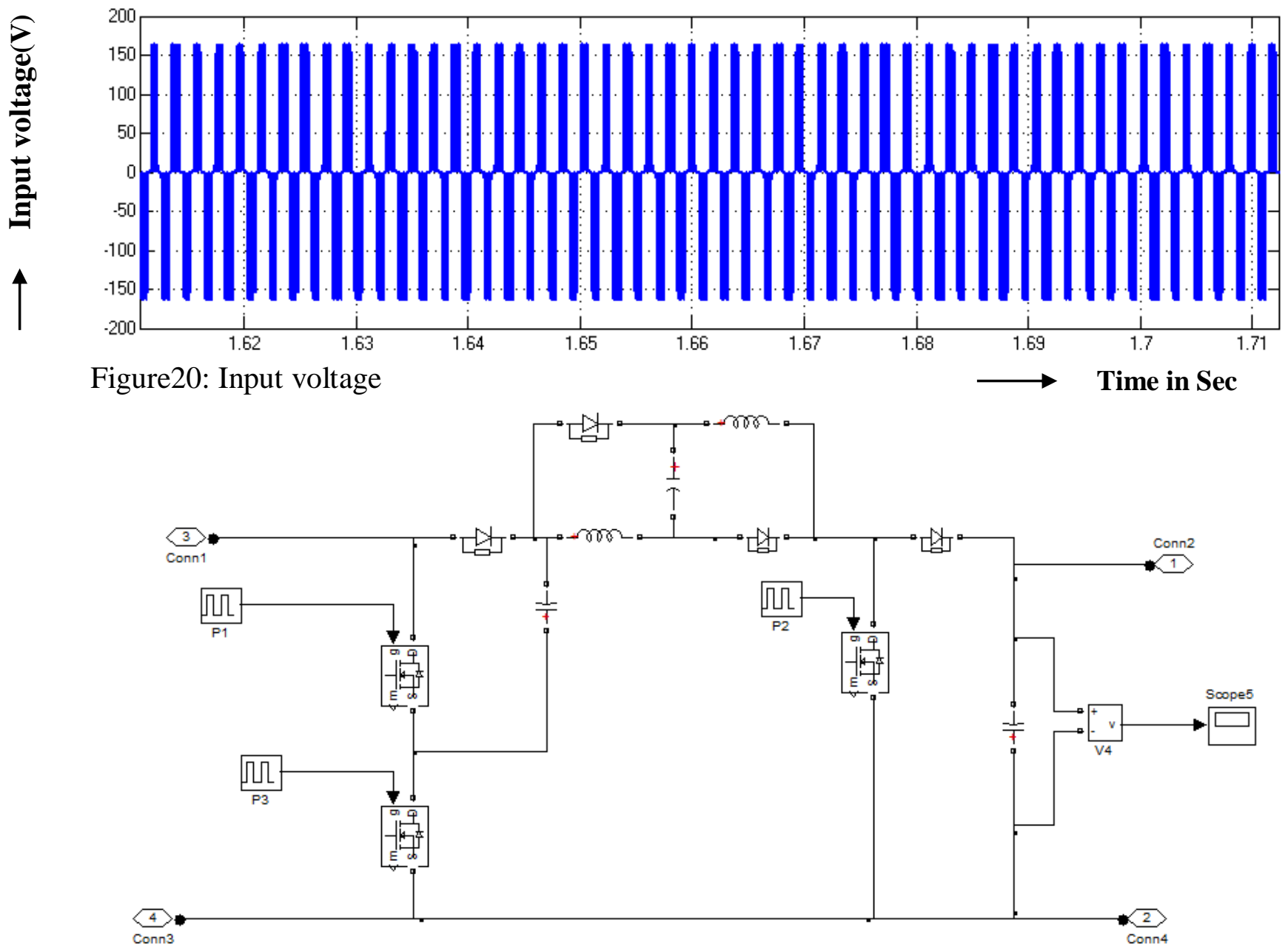

Figure21: Circuit diagram of bootstrap converter

The Voltage across bootstrap converter and the motor load are indicated in Figure 22 and 23. The Voltage across bootstrap converter is 480 Volts and Voltage across motor load value is 400 Volts.

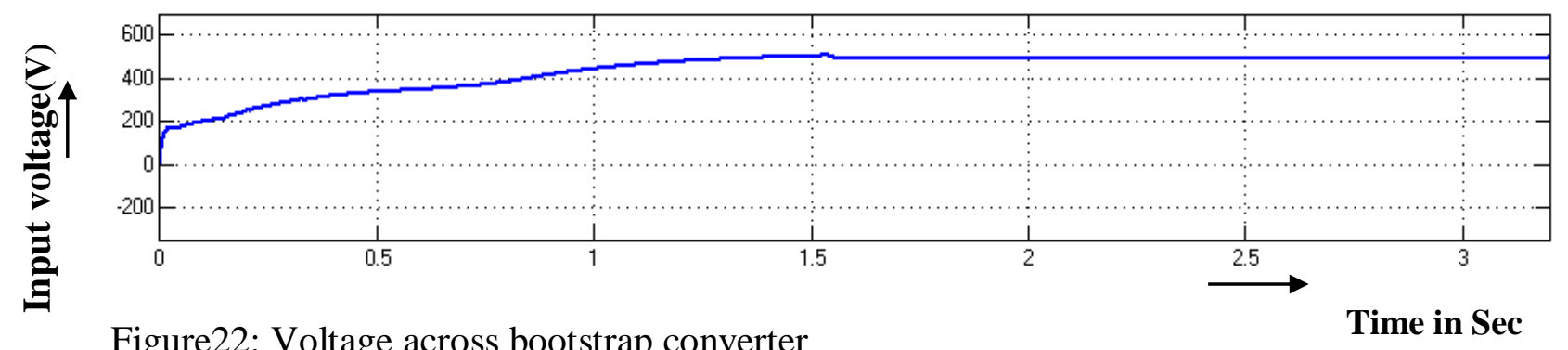

Figure22: Voltage across bootstrap converter 

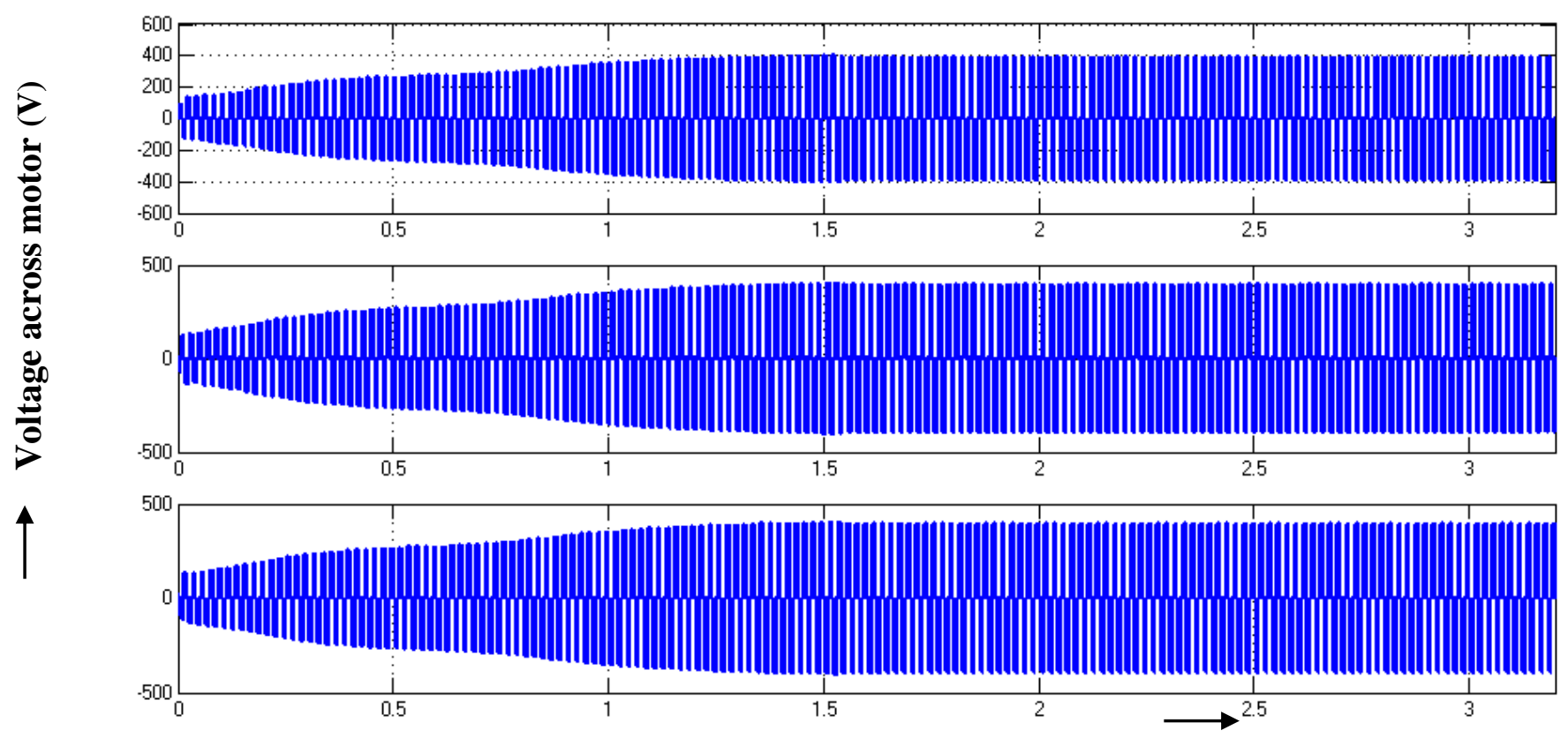

Figure23: Voltage across motor load

Time in Sec

Motor speedwith SVM inverter ofclosed two loopFOPID controller is 1290 RPM and it is sketched in Figure 24. Motor speed zoom out along with SVM inverter of closed two loopFOPID controller is 1290 RPM and is sketched in Figure 25. The Motor Torque is sketched in Figure 26.
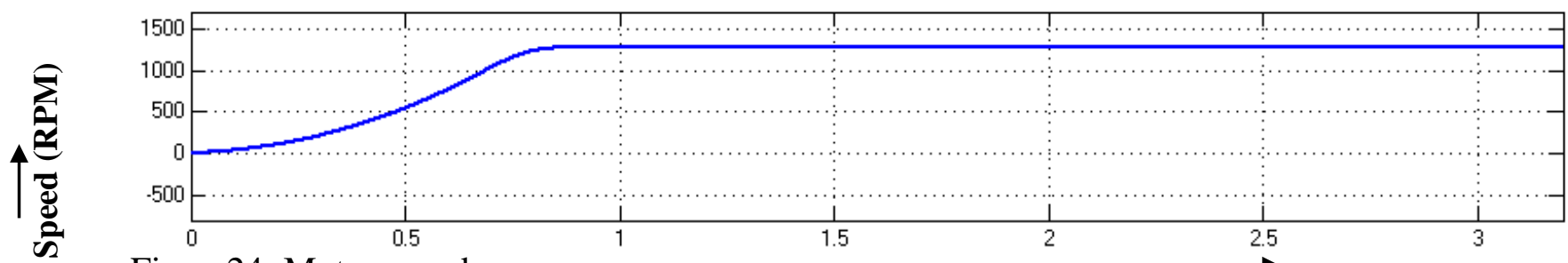

Figure24: Motor speed

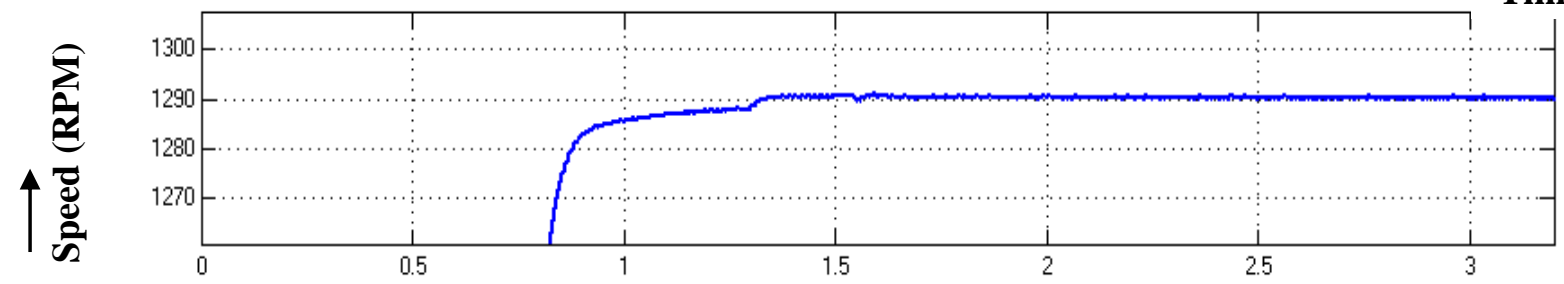

Figure25: Motor speeds zoom out

Time in Sec

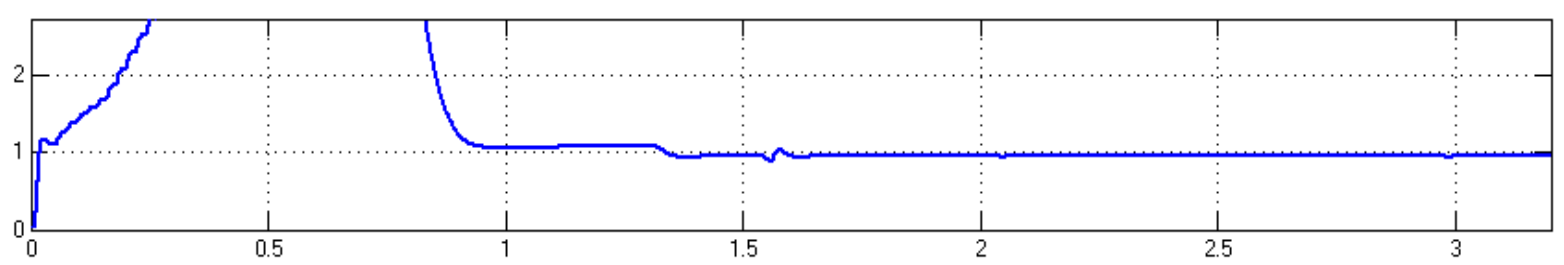

Figure26: Motor Torque

Time in Sec

Figure 27 outlines the BarChartof motor speed using PIC and $\mathrm{HC}$ along with its various TimeDomain Parameters. The table-1 explains the comparative relation of TimeDomainParameters for motor-speedusing PIC-HC and PR-PR, the 'Tr (rise-time)' is lessened from 1.34 
Sec to $1.32 \mathrm{Sec}$; 'Ts (Settling-time)' is lessened from $1.75 \mathrm{Sec}$ to $1.60 \mathrm{Sec}$; 'Tp (peak-time)' is lessenedfrom1.55 Sec to $1.47 \mathrm{Sec}$; 'Ess (Steady-stateerror)' is lessened from 0.5 RPM to 0.3 RPM.

Table-1

The comparative relation of Time Domain Parameters for motor speed using PI-HC and PRPR

\begin{tabular}{|l|l|l|l|l|}
\hline Controller & & & $\operatorname{Tp}(\mathrm{S}$ & Ess(RPM \\
\hline PR-PR & 1.34 & 1.75 & 1.55 & 0.5 \\
\hline PI-HC & 1.32 & 1.60 & 1.47 & 0.3 \\
\hline
\end{tabular}

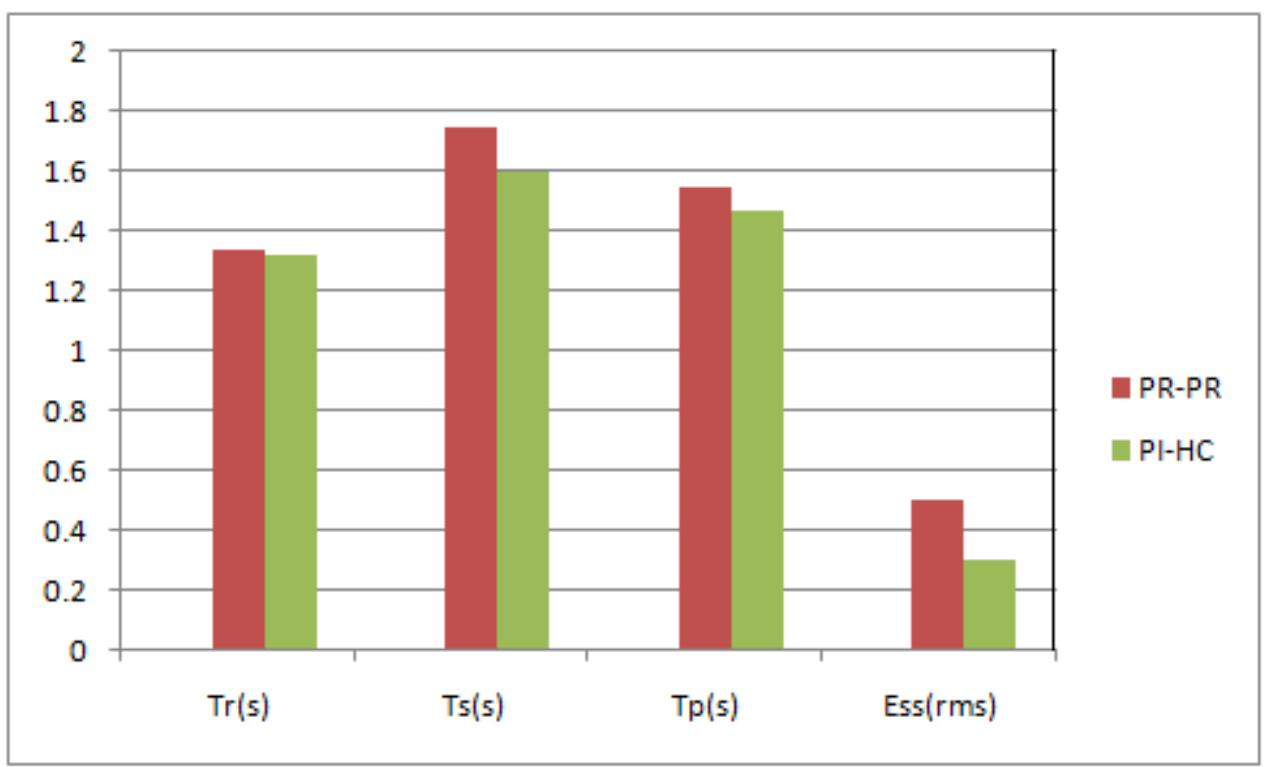

Figure 27.BarChartof Time Domain Parameters (Tr, Ts, Tp, Ess) for motor speed using PIC$\mathrm{HC}$ and PR-PR

Figure 28 outlines the BarChartof motor torque using PIC-HC and PR-PR along with its variousTimeDomainParameters. The table-2 explains the comparative relation of Time DomainParameters for motor-torque using PIC-HC and PR-PR.By using PIC-HC, the 'Tr (rise-time)' is lessened from 1.35 Sec to $1.33 \mathrm{Sec}$; 'Ts(Settling-time)' is lessened from 1.82 $\mathrm{Sec}$ to $1.67 \mathrm{Sec}$; 'Tp (peak-time)' is lessenedfrom 1.71 Sec to $1.67 \mathrm{Sec}$; 'Ess (Steady-stateerror)' is lessened from $0.3 \mathrm{~N}-\mathrm{m}$ to $0.1 \mathrm{~N}-\mathrm{m}$.

Table-2

The comparative relation of Time Domain Parameters for motor torque using PI-HC and PRPR

\begin{tabular}{|c|c|c|c|c|c|}
\hline Controller & $\operatorname{Tr}(\mathrm{s})$ & ) & $\operatorname{Ts}(\mathrm{s}$ & s) & $\begin{array}{c}\text { Ess( } \\
\mathrm{N}-\mathrm{m})\end{array}$ \\
\hline PR-PR & 1.35 & 2 & 1.8 & $1^{1.7}$ & 0.3 \\
\hline PI-HC & 1.33 & 7 & 1.6 & $9^{1.3}$ & 0.1 \\
\hline
\end{tabular}




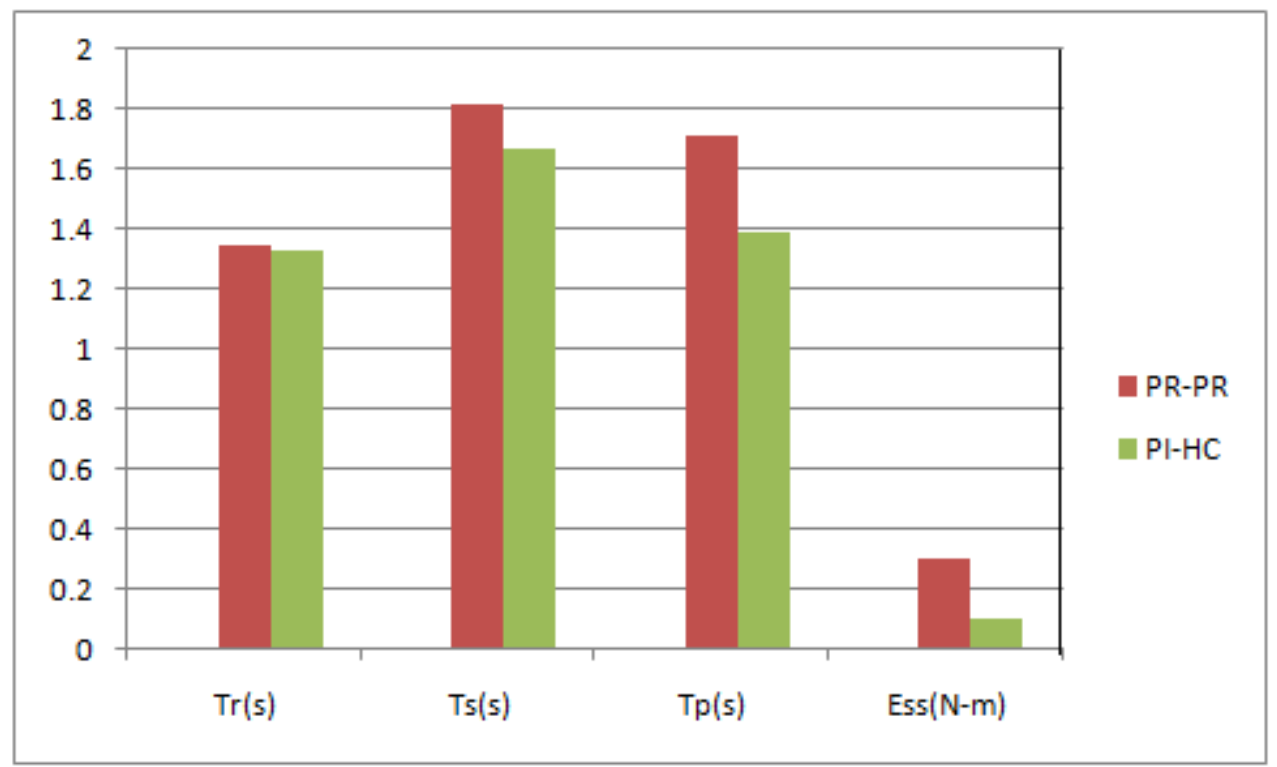

Figure 28.BarChartof Time Domain Parameters (Tr, Ts, Tp, Ess) for motor Torque using PI$\mathrm{HC}$ and PR-PR

\section{Experimental Results}

Hard-ware snap-shot of IM based Boot-strap converter with SVM invertersystem is sketched in Figur29. The hardware of BCSVMI consists of Motor load,inverter panel, BCB,control panel and RBC panel. The snap shot of the input voltage is outlined in Figure 30.

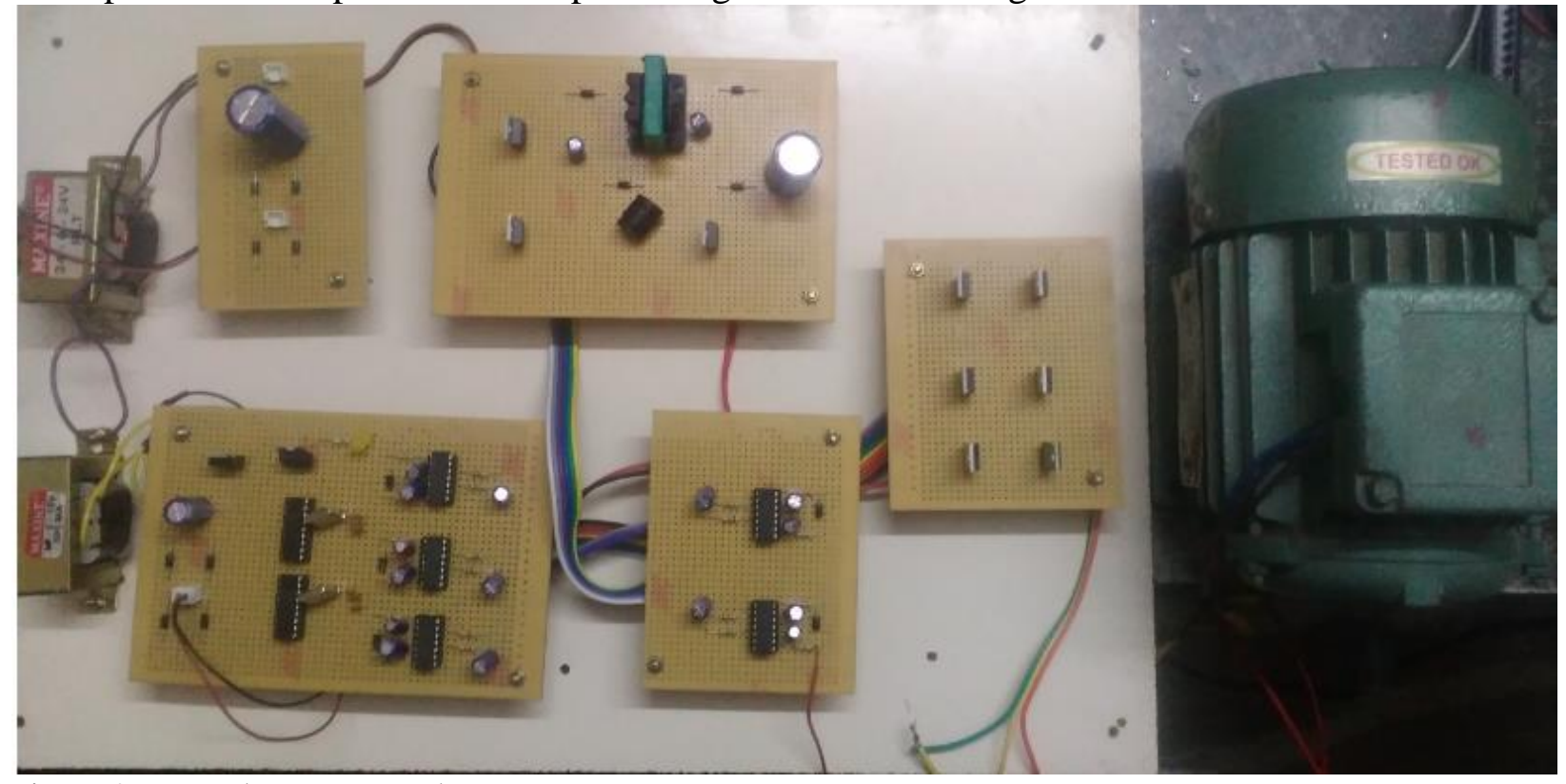

Figure29: Hardware snap shot

The Switching pulse for bootstrap converter across S1 and S2 is appeaed in Figure 30. 


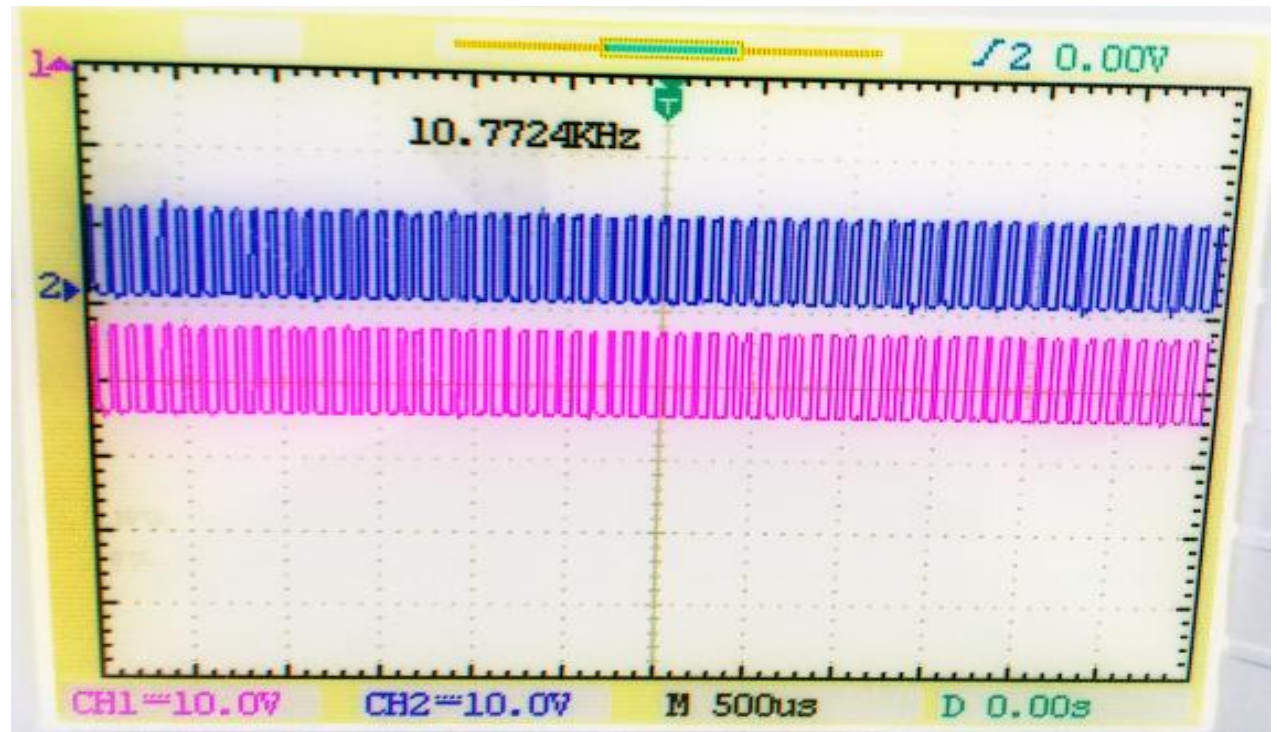

Figure 30: The Switching pulse for bootstrap converter across S1and S2,

Switching-pulse for inverter M1, M2and M3, M6are outlined in Figure 31 and Figure 32 respectively. From Figure 31 it is clear that its peak to peak value is increased.

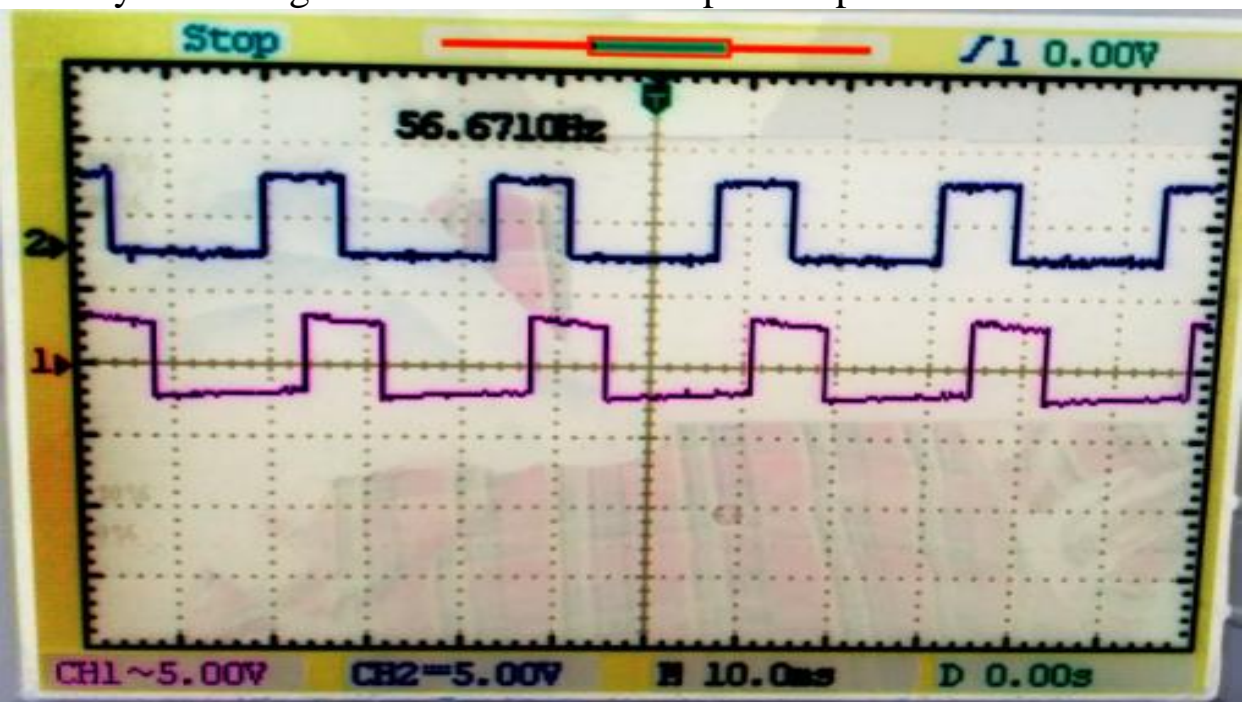

Figure31: Switching pulse for inverter M1,M2 


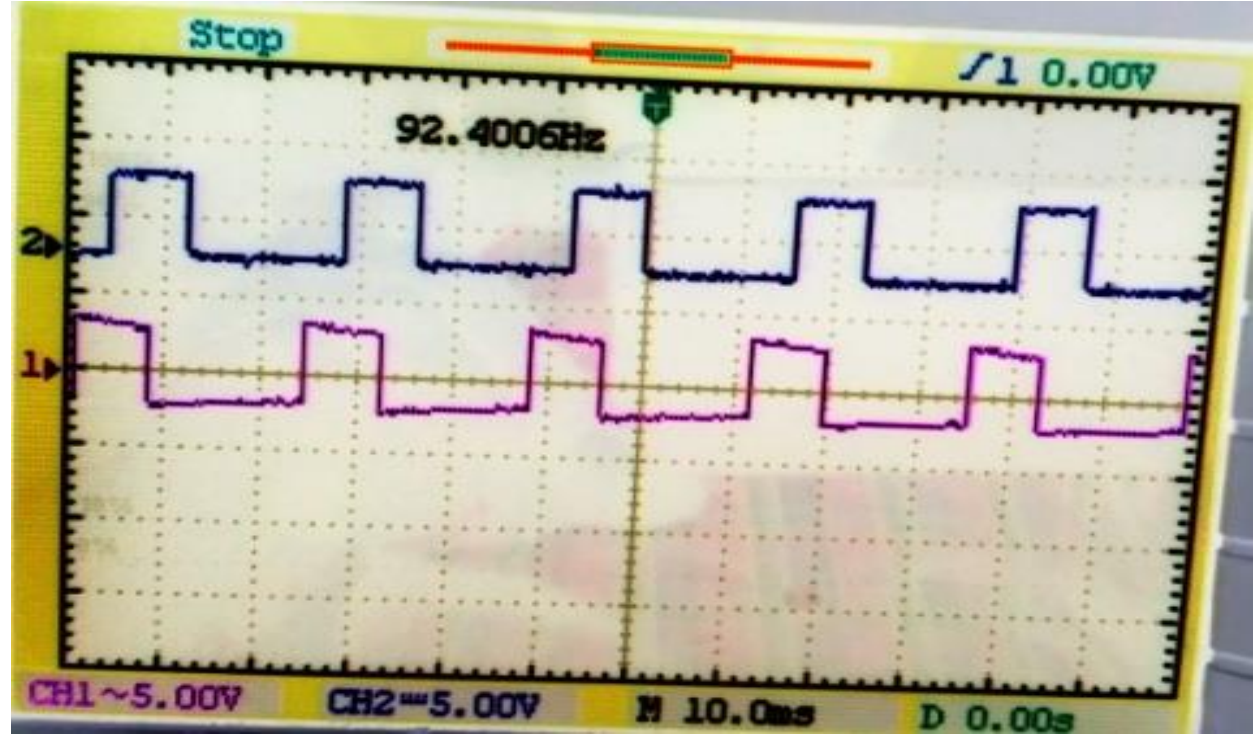

Figure32: Switching pulse for inverter M3,M6

The Motor load voltage and the motor current areoutlined in Figure 33 and 34 respectively.

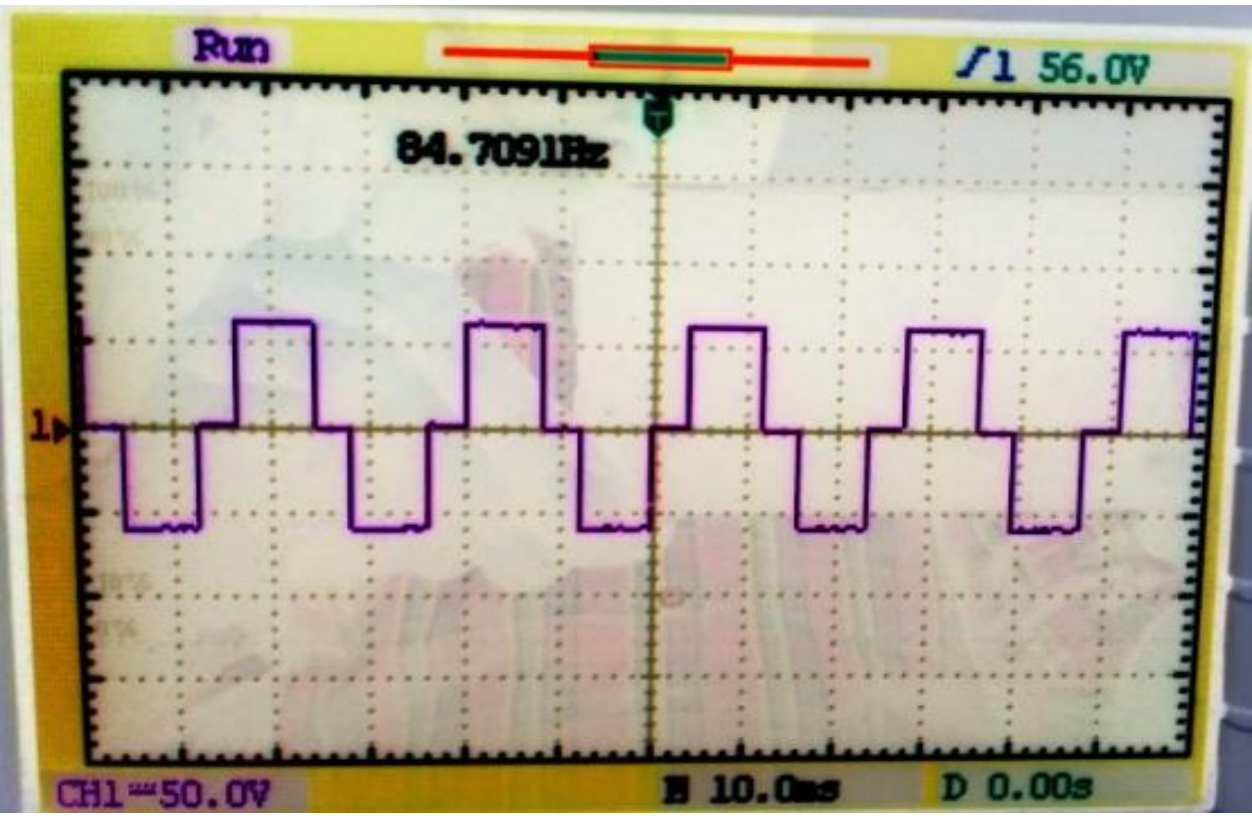

Figure33: The motor load voltage 


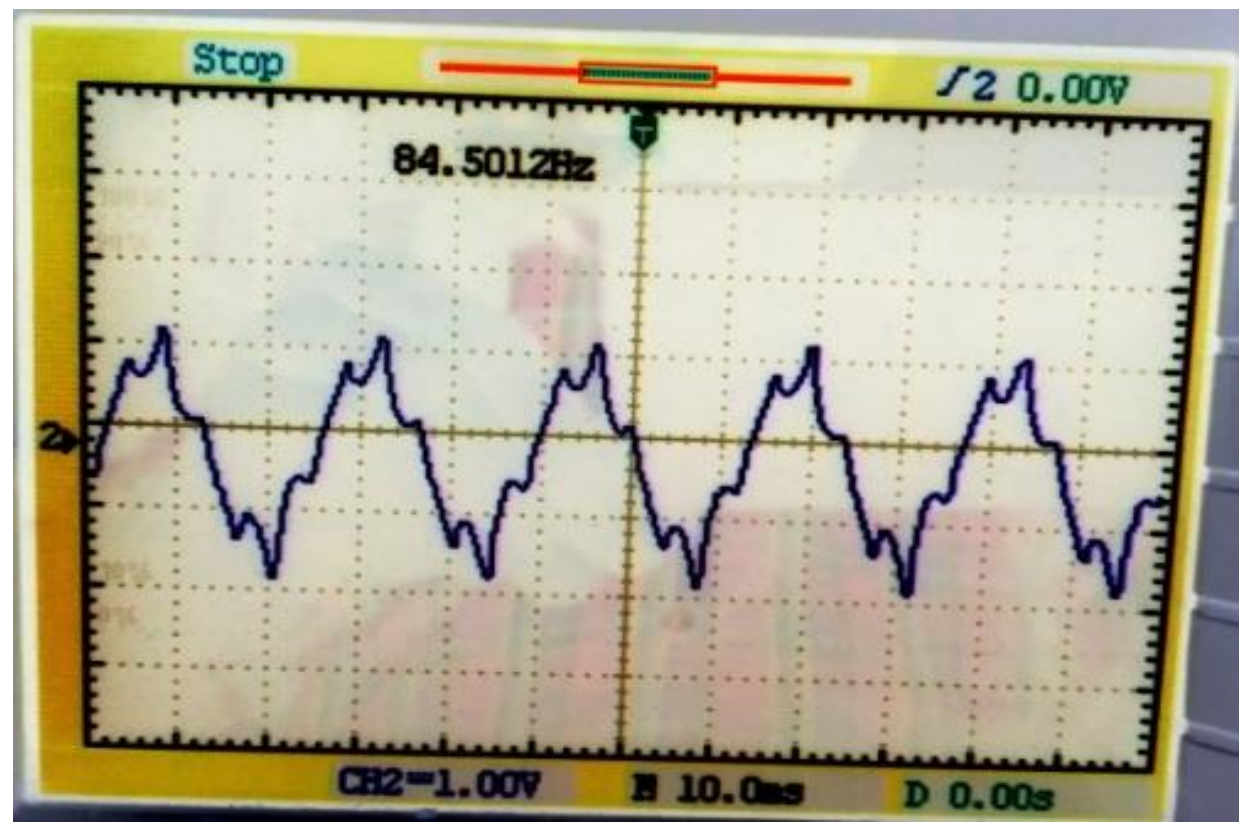

Figure34: The motor load current

\section{Conclusion}

The Closed two loop BSC- SVMI systems along with PR-PR and PI-HC are designed andsimulated using the simulation tool Mat-labSimulink. The outcomes of the simulations for the closed two loop systems with PR-PR and PI-HC are tabulated. The time domain parameters are obtained and the values are compared and represented using bar chart. The Ts(settling time) is reduced to $1.67 \mathrm{Sec}$ and Ess(steady state error) is reduced to $0.1 \mathrm{~N}-\mathrm{m}$ by using HC(Hysteresis controller). From the obtained response of outcome of PI-Hysteresis Controller system is better than thePR-PRcontrolled system. The hard-ware of BSC-SVMI is fabricatedand tested. The experimental results of SVMI have been presented for validation purpose. The proposed system has few benefits like fast responseandlow harmonics. The downside of bootstrap converter is that it is appropriate for low power. The presentwork brings out the performance of PI-hysteresis controlled BSC-SVMIIM. The investigations on Slide Mode controlled BSC-SVMIIM will be done in future.

\section{References}

[1]. YongKeun-Lee,JongKwangKim(2019), “Analysis of bootstrap circuit operation with an inverted PWM drive scheme for a three phase inverter for a brushless dc motor drive" Canadian Journal of Electrical and Computer Engineering ( Volum-42, Issue1, winter2019). 10.1109/CJECE.2019.2891850.

[2]. "DIPIPM application note bootstrap circuit design manual" in Dual in Line Package Intelligent Power Module, Tokyo, Japan: Mitsubishi ElectronicCorp, 2016.

[3]. K.Mainali, S.Madhusoodhanan, A. Tripathi, K.Vechalapu, A.De and S.Bhattacharya, "Design and evaluation of isolated gate driver power supply for medium voltage converter applications", Proc. IEEEAppl. Power Electron. Conf. Expo., pp1632-1639, 2016.

[4]. Ye, Zichao; Lei, Yutian; Liu, Wen Chuen; Shenoy, Pradeep S.; PilawaPodgurski, Robert Carl Nikolai (2019). Improved bootstrap methods for powering floating gate drivers of flying capacitor multilevel converters and hybrid switched capacitor converters. IEEE Transactions on PElectronics, 1-1. doi10.1109/TPEL.2019.2951116 
[5]. Y.Lei, W.Liu and RCN. PilawaPodgurski, "An analytical method to evaluate and design hybrid switched capacitor and multilevel converters", IEEE Trans. Power Electron., vol33,no.3,pp 2227-2240, Mar2018.

[6]. BZhu, SHu, GLiu, YHuang and XShe, " Low voltage stress buck boost converter with a high voltage conversion gain", IEEE Access, vol8,pp:95188-95196, May2020.

[7]. FPouladi, HFarzanehfard, EAdib and HLeSage, "Single switch soft switching LED driver suitable for battery operated systems", IEEE Trans. Ind. Electron., vol 66 no. 4, pp. 2726-2734Apr2019.

[8]. ShasanpourA, Baghramianand HMojallali, IEEE Trans. PElectron., vol35, no. 8, pp:8088-8101,Aug2020.

[9]. L.Jia, X.Sun, Z.Zheng, X.Ma and L.Dai, Multimode smooth switching strategy for eliminating the operational dead zone in non-inverting buck-boost converter",IEEE Trans. PElectron., vol35, no.3, pp. 3106-3113, Mar2020.

[10].GZhang, Yuan, S.SYu, NZhang, YWang and YZhang," Advanced-four-mode-modulation based four switch non inverting buck-boost converter with extra operation zone", IET-Power Electron., vol. 13,no. 10,pp. 2049-2059, Aug2020.

[11].SLiu, XXieand LYang, " Analysis modeling and implementation of a switching bi-directional buck-boost converter based on electric vehicle hybrid energy storage for V2G system", IEEE Access, vol 8, pp. 65868-65879,Apr2020.

[12]. FPouladi, HFarzanehfard and EAdib, "Battery operated soft switching resonant buck-boost LED driver with single magnetic element", IEEE Trans. PElectron., vol34, no.3, pp.2704-2711, Mar2019.

[13].HYJung, SHKim, BMoon and SHLee, " A new circuit design of two switch buck-boost converter",IEEEAccess, vol.6, pp. 47415-47423, Aug2018.

[14]. NZhang,

[15]. GZhang and KWSee, "Systematic derivation of dead zone elimination strategies for the non-inverting synchronous buck-boost converter", IEEE Trans Power Electron., vol. 33, no.,4, pp. 3497-3508,Apr2018.

[16].JWang, HWu, TYang, LZhang and YXing, " Bidirectional three phase DC-AC converter with embedded DC-DC converter and carrier based PWM strategy for wide voltage range applications", IEEE Trans. IElectron., vol. 66,no. 6, pp. 4144-4155, Jun2019.

[17]. YonghengYang, AKatherine, KimFredeBlaabjerg and AriyaSangwongwanich, Advances in Grid ConnectedPhotovoltaicPowerConversion Systems, Woodhead Publishing, Aug2018.

[18]. Geeta,; Aspalli,MS. [IEEE 2020 International conf., on power electronics \&iot applications in renewable energy and its control(PARC) Mathura, UttarPradesh, India(2020.2.28-2020.2.29)]2020Intern. Conf., onPElectronics\&IoT Apps inrenewableenergyanditscontrol(PARC)proportionalresonantcontrollerforsemiconverter-three phase VSI fedinductionmotordriveto enhancetimeresponse 169174. doi:10.1109/parc491932020236584.

[19].Premila,TR;KrishnaKumar,R (2019). Proportional resonant control of PV fed cascaded boost reboost inverter system. The International Journal of Electrical Engineering\& Education, 002072091988379, doi10.1177/0020720919883799.

[20]. Omrani, Siamak; Azizian, Mohammad Reza (2019). [ IEEE2019 Iranian Conference on RenewableEnergy\&Distributed Generation (ICREDG)Tehran, Iran (2019.6.11-2019.6.12)]2019, Iranian Conference on RenewableEnergy\&DistributedGeneration (ICREDG)PR FuzzyControl Improvement of Doubly Fed InductionGeneratorDuring Grid Faults. , 1-6. doi:10.1109/-ICREDG47187.2019.190165.

[21].GauriA.; Vinod, B.R.; ShinyG. (-2019). [IEEE 2019 International Conference on Electrical, Computer and CommunicationTechnologies (ICECCT) Coimbatore, India (2019.2.20-2019.2.22)] 2019 IEEE InternationalConferenceon Electrical, Computer and Communication Technologies(ICECCT)VectorControl Method for Induction Motor Drive Based on HysteresisControllerandPIControllerComparative Study,1-6. doi:10.1109/ICECCT.2019.8869497.

[22]. Talib, MHN.; IsaSN. Mat; HamidonHE.; IbrahimZ.; RasinZ. (2016). [IEEE2016 InternationalConference on Power and Ener. PECon) Melaka, Malaysia (2016.11.28-2016.11.29), IEEEInternational Conference on Power and Energy(PE Con) Hysteresis current control of induction motor drives using dSPACE DSP controller, pp. 522-527.doi10.1109/PECON.2016-7951617.

[23]. MdHairulNizamTalib, SitiNoormizaBintiMatIsa "Hysteresis current control of induction motor drives using dSPACE DSP controller" 2016, IEEE International Conferenceon Power and Energy(PECon), Nov.2016, DOI: 10.1109/PECON2016-7951617. 